\title{
Heavy Baryon Production and Decay
}

\author{
Isard Dunietz \\ Fermi National Accelerator Laboratory, P.O. Box 500, Batavia, IL 60510
}

(October 8, 2018)

\begin{abstract}
The branching ratio $B\left(\Lambda_{c} \rightarrow p K^{-} \pi^{+}\right)$normalizes the production and decay of charmed and bottom baryons. At present, this crucial branching ratio is extracted dominantly from $\bar{B} \rightarrow$ baryons analyses. This note questions several of the underlying assumptions and predicts sizable $\bar{B} \rightarrow D^{(*)} N \bar{N}^{\prime} X$ transitions, which were traditionally neglected. It predicts $B\left(\Lambda_{c} \rightarrow p K^{-} \pi^{+}\right)$to be significantly larger $(0.07 \pm 0.02)$ than the world average. Some consequences are briefly mentioned. Several techniques to measure $B\left(\Lambda_{c} \rightarrow p K^{-} \pi^{+}\right)$are outlined with existing or soon available data samples. By equating two recent CLEO results, an appendix obtains $B\left(D^{0} \rightarrow K^{-} \pi^{+}\right)=0.035 \pm 0.002$, which is somewhat smaller than the current world average.
\end{abstract}




\section{MOTIVATION}

Decays of heavy baryons allow novel tests of Heavy Quark Effective Theory (HQET) [1.2]. For instance, the structure of the $1 / m_{c}$ corrections is known for the semileptonic transition $\Lambda_{b} \rightarrow \Lambda_{c} \ell \nu$ [3]. That structure is theoretically simpler than the much studied $\bar{B} \rightarrow D^{(*)} \ell \nu$ one because the light degrees of freedom of the heavy baryon are spinless and isospinless, while those of the heavy meson are not. Heavy baryon decays allow one to refinef the extraction of the fundamental Cabibbo-Kobayashi-Maskawa (CKM) parameters [3.5.6] and could show CP violating effects [6]. Detailed studies of heavy baryons are thus important.

The mode $\Lambda_{c} \rightarrow p K^{-} \pi^{+}$plays a central role in those investigations, because of its sizable branching ratio and observability. Currently, most other $\Lambda_{c}$ branching ratios are normalized with respect to $B\left(\Lambda_{c} \rightarrow p K^{-} \pi^{+}\right)$. The branching fractions of other weakly decaying charmed baryons $\left(\Xi_{c}, \Omega_{c}\right)$ can also be tied to $B\left(\Lambda_{c} \rightarrow p K^{-} \pi^{+}\right)$. The importance of the $\Lambda_{c} \rightarrow p K^{-} \pi^{+}$ process is not limited to the charm sector but extends to the $b$-sector. Decay products of beautiful baryons will normally involve charmed baryons. Even bottom mesons decay non-negligibly into $\Lambda_{c}, \Xi_{c}, \Omega_{c}$ baryons.

The 1996 edition of the particle data group (PDG) quotes [7] $B\left(\Lambda_{c} \rightarrow p K^{-} \pi^{+}\right)=$ $0.044 \pm 0.006$, which is dominated by $\bar{B} \rightarrow$ baryons analyses. The "traditional" interpretation

of the most accurate and recent $\stackrel{(-)}{B} \rightarrow \Lambda_{c} X$ data [3] leads to a value of $B\left(\Lambda_{c} \rightarrow p K^{-} \pi^{+}\right)=$ $0.027 \pm 0.005$. Those "traditional" analyses have made simplifying assumptions which may not hold as discussed below. This note obtains a significantly larger

$$
B\left(\Lambda_{c} \rightarrow p K^{-} \pi^{+}\right)=0.07 \pm 0.02
$$

\footnotetext{
*The model-independent determination of $\left|V_{c b}\right|$ from inclusive semileptonic $\bar{B} \rightarrow X \ell \nu$ transitions involves corrections dependent on $\bar{\Lambda} \equiv m_{B}-m_{b}$ 画. While $\bar{\Lambda}$ is poorly known at present, it could be determined more accurately from the $\Lambda_{b} \rightarrow \Lambda_{c} \ell \nu$ process [3]. The theoretical input could thus be better controlled.
} 
by combining existing data with theory.

Section 2 discusses the derivation of this sizably larger branching ratio. Section 3 reviews the traditional extraction of $B\left(\Lambda_{c} \rightarrow p K^{-} \pi^{+}\right)$from $\bar{B} \rightarrow$ baryons analyses, and reviews the various employed assumptions. While the $\bar{B} \rightarrow D^{(*)} N \bar{N}^{\prime} X$ transitions were neglected, a straightforward theoretical Dalitz plot analysis shows that they probably are sizable [9]. Here $N^{\left({ }^{\prime}\right)}$ denotes a nucleon. This note predicts that

$$
B\left(\bar{B} \rightarrow D^{(*)} N \bar{N}^{\prime} X\right) \sim \text { few } \%
$$

and demonstrates that the assumption of neglecting $\bar{B} \rightarrow \Xi_{c} X, \Omega_{c} X$ cannot be justified. Finally, the so-called "model-independent" determination of [10,7]

$$
B(\bar{B} \rightarrow \text { baryons })=0.068 \pm 0.006
$$

is questionable as it neglected the $\bar{B} \rightarrow D^{(*)} N \bar{N}^{\prime} X$ processes. The latter part of Section 3 suggests several methods to search for and observe $\bar{B} \rightarrow D^{(*)} N \bar{N}^{\prime} X$ in existing data samples. The observation would put into further doubt the conventional $\bar{B} \rightarrow$ baryons model. It would necessitate a serious rethinking of how to accurately determine absolute branching ratios of heavy baryons. The goal of Section 4 is therefore to sketch several methods that are able to determine absolute $\Lambda_{c}$ branching ratios from existing or soon available data samples. Some implications of the significantly larger predicted $B\left(\Lambda_{c} \rightarrow p K^{-} \pi^{+}\right)$are discussed in Section 5. Section 6 concludes.

\section{II. $B\left(\Lambda_{c} \rightarrow p K^{-} \pi^{+}\right)$}

The 1996 particle data group value is [7]

$$
B\left(\Lambda_{c} \rightarrow p K^{-} \pi^{+}\right)=0.044 \pm 0.006
$$

It is dominated by $B \rightarrow$ baryons analyses, which the next section critically reviews. The recent and more accurate CLEO result [8] would imply a much reduced 


$$
B\left(\Lambda_{c} \rightarrow p K^{-} \pi^{+}\right)=0.027 \pm 0.005
$$

if one were allowed to use the conventional $B \rightarrow$ baryons analysis.

This note argues to use instead

$$
B\left(\Lambda_{c} \rightarrow p K^{-} \pi^{+}\right)=0.07 \pm 0.02
$$

which is obtained from 11

$$
B\left(\Lambda_{c} \rightarrow p K^{-} \pi^{+}\right)=\frac{B\left(\Lambda_{c} \rightarrow p K^{-} \pi^{+}\right)}{B\left(\Lambda_{c} \rightarrow \Lambda X \ell \nu\right)} \frac{B\left(\Lambda_{c} \rightarrow \Lambda X \ell \nu\right)}{B\left(\Lambda_{c} \rightarrow X_{s} \ell \nu\right)} \frac{\Gamma\left(\Lambda_{c} \rightarrow X_{s} \ell \nu\right)}{\Gamma\left(D^{0} \rightarrow X_{s} \ell \nu\right)} \frac{B\left(D^{0} \rightarrow X \ell \nu\right)}{\left(1+\left|\frac{V_{c d}}{V_{c s}}\right|^{2}\right)} \frac{\tau\left(\Lambda_{c}\right)}{\tau\left(D^{0}\right)}
$$

The various factors will be discussed in turn. Experiment informs us about [1]

$$
\frac{B\left(\Lambda_{c} \rightarrow p K^{-} \pi^{+}\right)}{B\left(\Lambda_{c} \rightarrow \Lambda X \ell \nu\right)}=1.93 \pm 0.10 \pm 0.33
$$

Because both the initial state $\Lambda_{c}$ and the $c \rightarrow s \ell \nu$ transition have zero isospin, the resulting final states are isospinless. Isospin symmetry gives

$$
\begin{gathered}
\Gamma\left(\Lambda_{c} \rightarrow n \bar{K}^{0} \ell \nu\right)=\Gamma\left(\Lambda_{c} \rightarrow p K^{-} \ell \nu\right), \\
\Gamma\left(\Lambda_{c} \rightarrow \Sigma^{+} \pi^{-} \ell \nu\right)=\Gamma\left(\Lambda_{c} \rightarrow \Sigma^{-} \pi^{+} \ell \nu\right)=\Gamma\left(\Lambda_{c} \rightarrow \Sigma^{0}[\rightarrow \Lambda X] \pi^{0} \ell \nu\right),
\end{gathered}
$$

and once applied to the ratio $f \equiv B\left(\Lambda_{c} \rightarrow \Lambda X \ell \nu\right) / B\left(\Lambda_{c} \rightarrow X_{s} \ell \nu\right)$ yields

$$
f=1 /\left(1+2 \frac{\Gamma\left(\Lambda_{c} \rightarrow \Sigma^{+} \pi^{-} \ell \nu\right)}{\Gamma\left(\Lambda_{c} \rightarrow \Lambda X \ell \nu\right)}+2 \frac{\Gamma\left(\Lambda_{c} \rightarrow p K^{-} \ell \nu\right)}{\Gamma\left(\Lambda_{c} \rightarrow \Lambda X \ell \nu\right)}\right)
$$

The underlying expectation is that the Cabibbo-allowed semileptonic transition $\Lambda_{c} \rightarrow$ $X_{s} \ell \nu$ consists almost entirely of $\Lambda X \ell \nu, \Sigma \pi \ell \nu$, and $N \bar{K} \ell \nu$. Further, the exclusive $\Lambda_{c} \rightarrow \Lambda \ell \nu$ transition is predicted to dominate (almost saturate) $\Lambda_{c} \rightarrow X_{s} \ell \nu$, in analogy to what has been observed in $D \rightarrow X_{s} \ell \nu$ processes. Cabibbo-allowed semileptonic $D$ decays are basically saturated by the exclusive $\bar{K} \ell \nu$ and $\bar{K}^{*} \ell \nu$ modes and no evidence for resonant $\bar{K}^{* *} \ell \nu$ or non-resonant $\bar{K} n \pi \ell \nu(n \geq 1)$ activity has been found [14]. Because the $\bar{K}^{(*)}$ analogue in the baryon sector is the $\Lambda$ hyperon, the observed $D$ decay pattern indicates a value close to 1 for 
$f$. Theoretical studies of invariant hadronic mass spectra in $\Lambda_{c} \rightarrow X_{s} \ell \nu$ transitions come to the same conclusion [12]. We thus estimate $f=0.9 \pm 0.1$. Fortunately, $f$ can be determined experimentally in the future via the right-hand-side of Eq. (2.5).

The ratio $r \equiv \Gamma\left(\Lambda_{c} \rightarrow X_{s} \ell \nu\right) / \Gamma\left(D^{0} \rightarrow X_{s} \ell \nu\right)$ has been estimated [13]

$$
r=1.3 \pm 0.2 .
$$

The prediction for $r$ to be larger than 1 follows from the operator-product-expansion formalism. The inclusive semileptonic $\Lambda_{c}$ and $D^{0}$ decay rates involve the same leading terms, but differ in the $\mathcal{O}\left(1 / m_{c}^{2}\right)$ corrections [13]. The most significant difference occurs in the average value of the spin energy. That value vanishes for the $\Lambda_{c}$, while it decreases $\Gamma\left(D \rightarrow X_{s} \ell \nu\right)$. That explains why $r$ is expected to be larger than 1 .

CLEO [14] gives the most precise $B\left(D^{0} \rightarrow X e \nu\right)=0.0664 \pm 0.0018 \pm 0.0029$ measurement to date and the lifetime ratio is taken from the PDG [7]. Eq. (2.4) expresses the branching ratio of $\Lambda_{c} \rightarrow p K^{-} \pi^{+}$in terms of Cabibbo favored transitions, because the $c \rightarrow d$ transitions of $\Lambda_{c}$ suffer from large Pauli interference enhancements [15] that are difficult to quantify. Those enhancements are absent for the semi-leptonic $D^{0}$ decays. While phase-space effects for the dominant exclusive transitions will change the ratio

$$
\Gamma\left(D^{0} \rightarrow X_{d} \ell \nu\right) / \Gamma\left(D^{0} \rightarrow X_{s} \ell \nu\right)
$$

away from the naive estimate $\left|V_{c d} / V_{c s}\right|^{2}$, the expected change will have negligible effect on the determination for $B\left(\Lambda_{c} \rightarrow p K^{-} \pi^{+}\right)$with present accuracy. Eq. (2.3) is obtained by combining the above.

\section{BARYON PRODUCTION IN $B$ DECAYS}

Because the dominant extractions of $B\left(\Lambda_{c} \rightarrow p K^{-} \pi^{+}\right)$[0, [8] involve $\bar{B} \rightarrow$ baryons analyses, it is worthwhile to review the various traditional assumptions made [16,10]. At the present level of accuracy, it is safe to neglect the $b \rightarrow u$ baryon producing transitions to obtain (see Fig. 1) 


$$
B(\bar{B} \rightarrow \text { baryons })=B\left(\bar{B} \rightarrow N_{c} X\right)+B\left(\bar{B} \rightarrow D^{(*)} N \bar{N}^{\prime} X\right)
$$

Here $N_{c}$ denotes any weakly decaying charmed baryon $\left(\Lambda_{c}, \Xi_{c}, \Omega_{c}\right), D$ denotes charmed mesons and $N^{\left({ }^{\prime}\right)}$ stands for a nucleon. The $\bar{B} \rightarrow D^{(*)} N \bar{N}^{\prime} X$ processes were traditionally neglected, because of arguments based on phase space suppression [16,10]. One assumed that

$$
B(\bar{B} \rightarrow \text { baryons })=B\left(\bar{B} \rightarrow N_{c} X\right)
$$

Since at the time neither $\Xi_{c}$ nor $\Omega_{c}$ production in $\bar{B}$ decays were observed, they were neglected. One thus obtained

$$
B(\bar{B} \rightarrow \text { baryons })=B\left(\bar{B} \rightarrow \Lambda_{c} X\right) .
$$

This report distinguishes flavor-specific branching fractions $-B(\bar{B} \rightarrow T X)$ and $B(\bar{B} \rightarrow$ $\bar{T} X)$-from the flavor-blind yield per $B$ decay

$$
Y_{T} \equiv B(\bar{B} \rightarrow T X)+B(\bar{B} \rightarrow \bar{T} X)
$$

where $\bar{B}$ represents a weighted average of $B^{-}$and $\bar{B}^{0}$. From flavor-specific and flavor-blind light baryon production in $B$ meson decays $[\stackrel{(-)}{B} \rightarrow p, p \bar{p}, \Lambda, \Lambda \bar{p}, \Lambda \bar{\Lambda}]$, one deduced that [10]

$$
B(\bar{B} \rightarrow \text { baryons })=0.068 \pm 0.006
$$

The $B\left(\bar{B} \rightarrow \Lambda_{c} X\right) B\left(\Lambda_{c} \rightarrow p K^{-} \pi^{+}\right)$measurement was then used to obtain $B\left(\Lambda_{c} \rightarrow p K^{-} \pi^{+}\right)$ by substituting $B\left(\bar{B} \rightarrow \Lambda_{c} X\right)$ by the "measured" $B(\bar{B} \rightarrow$ baryons) [Eq. (3.5)]. The most accurate measurement to date is [8]

$$
Y_{\Lambda_{c}} \times B\left(\Lambda_{c} \rightarrow p K^{-} \pi^{+}\right)=(1.81 \pm 0.22 \pm 0.24) \times 10^{-3}
$$

from which Eq. (2.2) is obtained. That summarizes the traditional understanding of baryon production in $B$ meson decays and the conventional determination of $B\left(\Lambda_{c} \rightarrow p K^{-} \pi^{+}\right)$. 


\section{A. Critique}

In contrast, our picture of baryon yields in $B$ meson decays is more involved, and $B\left(\Lambda_{c} \rightarrow\right.$ $\left.p K^{-} \pi^{+}\right)$is significantly larger than currently believed. First, are the $\bar{B} \rightarrow D^{(*)} N \bar{N}^{\prime} X$ transitions really negligible? A straightforward theoretical Dalitz plot analysis of the quark subprocess indicates that they probably are sizable. Simple accounting of the various baryon yields leads independently to the same conclusion. Thus, the traditional assumption [Eq. (3.2)] is probably not justified. Second, is it permissible to neglect $\Xi_{c}, \Omega_{c}$ production in $\bar{B}$ meson decays [Eq. (3.3)]? Clearly not, since $\bar{B} \rightarrow \Xi_{c}$ has been observed [20 22], and $B\left(\bar{B} \rightarrow \Xi_{c} X, \Omega_{c} X\right)$ has been predicted [9] to be a sizable fraction with respect to $B\left(\bar{B} \rightarrow \Lambda_{c} X\right)$

Third, the inclusive $B(\bar{B} \rightarrow$ baryons) determination [Eq. (3.5)] assumed Eq. (3.2). Because that assumption is probably not justified, the result [Eq. (3.5)] inferred from flavor-specific light baryon yields is questionable. Instead of the traditional extraction of $B\left(\Lambda_{c} \rightarrow p K^{-} \pi^{+}\right)$, the measurement [Eq. (3.6)] is used to determine the flavor-blind $\Lambda_{c}$ yield in $B$ decays,

$$
Y_{\Lambda_{c}}=(0.026 \pm 0.005) \frac{0.07}{B\left(\Lambda_{c} \rightarrow p K^{-} \pi^{+}\right)}
$$

Only about $2.6 \%$ of all $\bar{B}$ decays are seen in modes involving $\stackrel{(-)}{\Lambda}_{c}$, in contrast to conventional belief [10,16, [1, 17]. Before constructing a consistent view of baryon production in $B$ decays, two apparently puzzling observations are reviewed:

(a) The momentum spectrum of produced $\Lambda_{c}$ in $\bar{B}$ decays is very soft 8 .

(b) The two-body modes $\bar{B} \rightarrow\left\{\Lambda_{c}, \Sigma_{c}\right\}\{\bar{p}, \bar{\Delta}\}$, shown in Figure 2, have not been observed. Only tight upper limits at the $10^{-3}$ level exist [7, 18].

\section{B. Previous attempt to solve the puzzles}

To resolve these puzzles it was hypothesized that baryon production in $B$ decays is governed by the $b \rightarrow c \bar{c} s$ transition (see Fig. 3) [19]. The $\bar{\Lambda}_{c}$ momentum spectrum is 
soft because the $\bar{\Lambda}_{c}$ 's are produced in association with the heavy $\Xi_{c}^{(r)}$, where superscript " $r$ " denotes resonance. The two-body modes $\bar{B} \rightarrow\left\{\Lambda_{c}, \Sigma_{c}\right\}\{\bar{p}, \bar{\Delta}\}$ are naturally absent. Further, this mechanism gives rise to "wrong-sign" $b \rightarrow \bar{\Lambda}_{c}$ transitions in contrast to the conventional "right-sign" $b \rightarrow \Lambda_{c}$ processes. Finally, it followed that $\Xi_{c}$ production in $\bar{B}$ decays is large and not negligible as commonly assumed.

Subsequently, CLEO found evidence for a large $\Xi_{c}$ yield [21,20]

$$
Y_{\Xi_{c}}=0.039 \pm 0.015
$$

That same analysis measured the "wrong-sign" to "right-sign" $\Lambda_{c}$ production in $B$ meson decays to be small [20]

$$
r_{\Lambda_{c}} \equiv \frac{B\left(\bar{B} \rightarrow \bar{\Lambda}_{c} X\right)}{B\left(\bar{B} \rightarrow \Lambda_{c} X\right)}=0.20 \pm 0.14
$$

This result indicated that the $\bar{B} \rightarrow \Xi_{c}^{(r)} \bar{\Lambda}_{c} X$ processes are not dominant, and refuted the hypothesis that baryon production in $B$ decays is dominated by the $b \rightarrow c \bar{c} s$ transition.

The flavor-specific $\Xi_{c}$ and $\Omega_{c}$ production in $\stackrel{(-)}{B}$ meson decays can be correlated to the much more accurately measured flavor-specific $\Lambda_{c}$ yields [9]. For a full list of predictions, please consult Ref. [9]. One prediction is that

$$
\frac{Y_{\Xi_{c}}}{Y_{\Lambda_{c}}}=0.38 \pm 0.10
$$

and once combined with (3.7) predicts that

$$
Y_{\Xi_{c}}=(0.010 \pm 0.003) \frac{0.07}{B\left(\Lambda_{c} \rightarrow p K^{-} \pi^{+}\right)}=0.010 \pm 0.004 .
$$

The much larger central value quoted by CLEO (3.8) indicates that the absolute branching ratio scale of $\Xi_{c}$ decays is in truth much larger than assumed. Theoretical support can be obtained from a recent paper of Voloshin [15]. Because of the above reasons, the CLEO collaboration now cites [22]

$$
Y_{\Xi_{c}}=0.020 \pm 0.010
$$

There remains little doubt that $\Xi_{c}$ production is sizable in $\bar{B}$ decays. Thus the determination of $B\left(\Lambda_{c} \rightarrow p K^{-} \pi^{+}\right)$from previous $\bar{B} \rightarrow$ baryons analyses is questionable. 


\section{Towards a consistent view of baryon production in $B$ decays}

Puzzles (a) and (b) can be explained by noting that a straightforward Dalitz plot for the dominant $b \rightarrow c \bar{u} d$ transition predicts the $c d$ invariant mass to be very large [9]. (The predicted invariant $c d$ mass distribution follows from the $V-A$ nature of the $b \rightarrow c \bar{u} d$ process.) If the $c d$ forms a charmed baryon [Figure 1], then in general this baryon will be significantly more massive than a $\Lambda_{c}$ or $\Sigma_{c}$, which explains puzzle (b). Further, such very massive $c d q$ objects or highly excited charmed baryon resonances would be seen usually as $\Lambda_{c} n \pi(n \geq 1)$. That explains naturally the observed soft $\Lambda_{c}$ momentum spectrum [puzzle (a)].

Analogously the invariant $c s$ mass in $b \rightarrow c s \bar{c}$ transitions is predicted to be very high. The $\Xi_{c}^{r}$ produced in $\bar{B} \rightarrow \Xi_{c}^{(r)} \bar{\Lambda}_{c} X$ processes could be seen significantly as $\Lambda_{c} \bar{K} X[\Lambda D X]$ which would lead to $\bar{B} \rightarrow \Lambda_{c} \bar{\Lambda}_{c} \bar{K} X\left[\Lambda D \bar{\Lambda}_{c} X\right]$ transitions. Such transitions could comprise a non-negligible fraction of the inclusive $\Lambda_{c}$ production in $\bar{B}$ decays and could show up as $\Lambda_{c} \bar{\Lambda}$ correlations in single $\stackrel{(-)}{B}$ decays."

While the theoretical Dalitz plot argument predicts the initially produced charmed baryons (via $b \rightarrow c$ ) to be highly excited, this is not expected of their pair produced antibaryons (via $b \rightarrow \bar{u}$ or $b \rightarrow \bar{c}$ ). The $V-A$ nature of the interaction favors smaller energies for the $\bar{u}$ or $\bar{c}$ antiquark in the restframe of the decaying $b$. Since the spectator antiquark $\bar{q}_{s}$ of the $\bar{B}\left(\equiv b \bar{q}_{s}\right)$ meson involves only a modest Fermi momentum, the invariant mass of the $\bar{u} \bar{q}_{s}$ or $\bar{c} \bar{q}_{s}$ system is also expected to be modest.

The very massive $c d q$ produced in $b \rightarrow c d \bar{u}$ transitions could be seen sizably as $D^{(*)} N X$. The $\bar{B}$ meson could be seen therefore in $\bar{B} \rightarrow D^{(*)} N \bar{N}^{\prime} X$ processes, in contrast to prevailing belief. Figure 4 shows another $\bar{B} \rightarrow D^{(*)} N \bar{N}^{\prime} X$ amplitude where the virtual $W^{-} \rightarrow \bar{u} d$

\footnotetext{
${ }^{\dagger}$ If $\Lambda_{c} \bar{\Lambda}_{c}$ production in $B$ decays turns out to be sizable, then the statement of Ref. 16] that their $B\left(\Lambda_{c} \rightarrow p K^{-} \pi^{+}\right)$measurement should be considered strictly as a lower limit has to be modified.
} 
hadronizes into a light baryon antibaryon pair.f Sizable $\bar{B} \rightarrow D^{(*)} N \bar{N}^{\prime} X$ processes would invalidate the assumption that baryon production involves, in general, weakly-decaying charmed baryons (3.2). The current determinations of $B\left(\Lambda_{c} \rightarrow p K^{-} \pi^{+}\right)$from $\bar{B} \rightarrow$ baryons analyses would have to be modified.

Another reason why the $\bar{B} \rightarrow D^{(*)} N \bar{N}^{\prime} X$ processes were neglected is the tight upper limit 16]

$$
B\left(\bar{B} \rightarrow D^{*+} p \bar{p} X\right)<0.4 \%
$$

Our scenario survives, however, because of flavor-correlations [9]. Consider the very massive $c d q$ object. It could be seen as a $D^{(*)+}$, which would normally not be produced in association with a $p$, because

$$
c d q \rightarrow(c \bar{d})(d d q)=D^{(*)+}\left\{n, \Delta^{0,-}, \ldots\right\} .
$$

If a $p$ is required in the final state, it is more readily correlated with a $D^{(*) 0}$ from $c d q$ decays. The virtual $W^{-} \rightarrow \bar{u} d$ normally hadronizes as $\bar{u} d \rightarrow \bar{p} n, \ldots$ and may thus survive the constraint of Eq. (3.11). This note predicts that

$$
B\left(\bar{B} \rightarrow D^{(*)} N \bar{N}^{\prime} X\right) \sim \text { few } \%
$$

from $B\left(\bar{B} \rightarrow D^{(*)} N \bar{N}^{\prime} X\right)=B(\bar{B} \rightarrow$ baryons $)-B\left(\bar{B} \rightarrow N_{c} X\right)$. First we discuss what can be inferred about inclusive baryon production in $B$ decays, and then we determine $B\left(\bar{B} \rightarrow N_{c} X\right)$ [9]. Prediction (3.12) follows.

$$
\text { D. } B(\bar{B} \rightarrow \text { baryons })
$$

The "accepted" value [7.10],

$$
B(\bar{B} \rightarrow \text { baryons })=0.068 \pm 0.006
$$

\footnotetext{
$\ddagger$ The size of this amplitude can be estimated from baryon production measurements at $e^{+} e^{-}$ colliders at c.m. energies $\sqrt{s}$ that satisfy $2 m_{p}<\sqrt{s}<m_{B}-m_{D}$.
} 
is obtained from flavor-specific, light baryon yields,

$$
\stackrel{(-)}{B} \rightarrow p, p \bar{p}, \Lambda, p \bar{\Lambda}, \Lambda \bar{\Lambda}
$$

under the assumption that baryon production always involves an $N_{c}$ [Eq. (3.2)]. The assumption probably does not hold, raising the question about the accurate value for $B(\bar{B} \rightarrow$ baryons). Model-independent lower limits can be derived from the light baryon measurements 3

$$
\begin{aligned}
B(\bar{B} \rightarrow \text { baryons }) & \geq \operatorname{Max}\left\{\frac{Y_{p}}{2}, \frac{Y_{\text {direct } p}+Y_{\Lambda}}{2}, Y_{p}-B(\bar{B} \rightarrow p \bar{p} X)\right\}= \\
& =\operatorname{Max}\{0.040 \pm 0.002,0.0475 \pm 0.0035,0.055 \pm 0.005\}
\end{aligned}
$$

Here the flavor-blind yield is defined in Eq. (3.4), and the values summarized in Ref. [7] were used.

Isospin arguments 23 could be used to determine $B(\bar{B} \rightarrow$ baryons $)$. Consider the dominant baryon producing transition $b \rightarrow c \bar{u} d$. The $\bar{B} \rightarrow N$ transition can proceed in several ways, some of which violate isospin even after the weak decay of the $\bar{B}$ (such as the $\bar{B} \rightarrow N_{c} \rightarrow N$ cascades). Thus, we focus instead on the light antibaryon yield in $\bar{B}$ meson decays, $\bar{B} \rightarrow \bar{N}^{\prime}$. Because this $\bar{N}^{\prime}$ "contains" the $\bar{u}$ from the $b \rightarrow c \bar{u} d$ transition and the other antiquarks forming the $\bar{N}^{\prime}$ are as likely to be a $\bar{d}$ as a $\bar{u}$, we expect more $\bar{p}$ than $\bar{n}$ production. Suppose that the ratio of $\bar{p} / \bar{n}$ production falls within the range

$$
1<\frac{B(\bar{B} \rightarrow \bar{p} X)}{B(\bar{B} \rightarrow \bar{n} X)}<3 .
$$

That range combined with the measurement of [10

$$
\begin{gathered}
B(\bar{B} \rightarrow \bar{p} X)=0.048 \pm 0.004, \text { yields } \\
0.097 \pm 0.009>B(\bar{B} \rightarrow \text { baryons })>0.065 \pm 0.006 .
\end{gathered}
$$

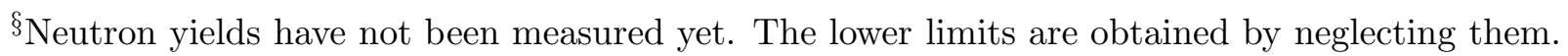


In summary, the inclusive baryon yield is in excess of 0.05 [Eq. (3.15)], and probably somewhere in the 0.06 - 0.10 range. Studies of neutron yields in $B$ decays offer one modelindependent way to determine $B(\bar{B} \rightarrow$ baryons $)$.

\section{E. $N_{c}$ production in $\bar{B}$ decays}

Once the value of $B\left(\bar{B} \rightarrow N_{c} X\right)$ is established, the $\bar{B} \rightarrow D^{(*)} N \bar{N}^{\prime} X$ fraction can be determined. The $B\left(\bar{B} \rightarrow N_{c} X\right)$ is determined in two steps,

$$
B\left(\bar{B} \rightarrow N_{c} X\right)=B\left(\bar{B} \rightarrow \Lambda_{c} X\right)+\left[\left(B\left(\bar{B} \rightarrow \Xi_{c} X\right)+B\left(\bar{B} \rightarrow \Omega_{c} X\right)\right]\right.
$$

The flavor-specific $B\left(\bar{B} \rightarrow \Lambda_{c} X\right)$ is taken from experiment [8,20], whereas $B\left(\bar{B} \rightarrow \Xi_{c} X\right)$

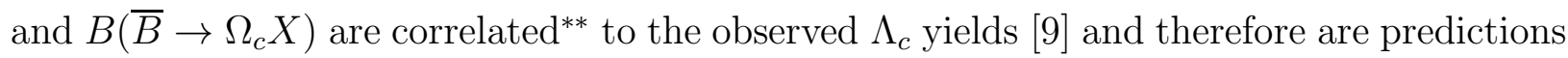
dependent on $B\left(\Lambda_{c} \rightarrow p K^{-} \pi^{+}\right)$,

$$
B\left(\bar{B} \rightarrow N_{c} X\right)=(0.032 \pm 0.006) \frac{0.07}{B\left(\Lambda_{c} \rightarrow p K^{-} \pi^{+}\right)}=0.032 \pm 0.011
$$

The last equation follows by inserting $0.07 \pm 0.02$ for $B\left(\Lambda_{c} \rightarrow p K^{-} \pi^{+}\right)$. Since the inclusive baryon yield is at the 0.06-0.1 level and $B\left(\bar{B} \rightarrow N_{c} X\right)$ is given by (3.20), we predict that probably

$$
B\left(\bar{B} \rightarrow D^{(*)} N \bar{N}^{\prime} X\right) \sim \text { few } \%
$$

Our prediction gets additional support from the theoretical Dalitz plot argument outlined above [9]. Table 1 summarizes this section. The existing data samples are sufficiently large to search for and observe sizable $\bar{B} \rightarrow D^{(*)} N \bar{N}^{\prime} X$ processes.

\footnotetext{
**This note does not use the direct $\Xi_{c}$ measurement in $\bar{B}$ decays, because of the very large uncertainties involved (see above). Instead, the uncertainties are drastically reduced by correlating the predicted $\Xi_{c}$ and $\Omega_{c}$ yields to the better measured $\Lambda_{c}$ yield.
} 


\section{F. $\bar{B} \rightarrow D^{(*)} N \bar{N}^{\prime} X$ Search}

Here we list a few suggestions to search for $\bar{B} \rightarrow D^{(*)} N \bar{N}^{\prime} X$. At the $\Upsilon(4 S)$ one could look for non-trivial angular correlations between a $D^{(*)}$ and a light baryon or antibaryon $\stackrel{(-)}{N}$. If the $D^{(*)}$ comes from one $\bar{B}$ and the $\stackrel{(-)}{N}$ from the other $B$, then they should be distributed almost isotropically 24]. On the other hand, observing a nontrivial angular correlation would indicate single $\bar{B}$ parentage. It is likely, however, that either the $D^{(*)}$ or the $\stackrel{(-)}{N}$ or both are soft, in which case the angular correlations are largely lost. Thus we recommend to search for the triple correlation $\ell^{+} D^{(*)} \stackrel{(-)}{N}$ on the $\Upsilon(4 S)$ [9]. The $\ell^{+}$and the $D^{(*)}$ cannot originate from a single $\stackrel{(-)}{B}$ meson, because of their flavors. Either the $\stackrel{(-)}{N}$ and the lepton share $B$ parentage or the $\stackrel{(-)}{N}$ and the $D^{(*)}$ originate from the same $\bar{B}$. While the latter interpretation is our coveted signal, the former is very unlikely. The former interpretation would indicate semileptonic $B$ decay in conjunction with baryon/antibaryon production

$$
B \rightarrow \bar{N}_{c} N^{\prime} \ell^{+} \nu
$$

which is expected theoretically to be tiny, and for which tight upper limits already exist [7.25.

Bottom hadrons produced at $Z^{0}$ factories are boosted and hadronize generally in opposing hemispheres. After selecting a $b$-enriched event, one could search, in a single $b$ hemisphere, for the predicted few percent (detached) $D^{(*)} \bar{N}$ correlation.

At either $e^{+} e^{-}$or hadron colliders, one may attempt to reconstruct the $\bar{B} \rightarrow D^{(*)} N \bar{N}^{\prime} \pi^{\prime}$ s

\footnotetext{
${ }^{\dagger \dagger} \mathrm{A}$ sufficiently large lepton momentum removes any background from $\bar{B} \rightarrow \Xi_{c} \bar{\Lambda}_{c} X$, where one of the charmed baryons decays semileptonically and either one contributes the $\stackrel{(-)}{N}$.

$\ddagger \ddagger$ The small $(\sim 0.5 \%)$ background from $\bar{\Lambda}_{b} \rightarrow \bar{N}_{c}[\rightarrow \bar{N} X] D^{(*)} K X \Longrightarrow \bar{N} D^{(*)}$ processes can be significantly reduced by (a) flavor-tagging, which enriches $b$ over $\bar{b}$ content, (b) enhancing $B^{-}$ parentage via vertex charge, which reduces the $b$-baryon background thereby making even the (detached) $D^{(*)} N$ correlations a convincing signal.
} 
modes. The existence of a $\stackrel{(-)}{n}$ can be inferred in analogy to methods developed for $\nu$ or $K_{L}$ reconstruction. Sizable $\bar{B} \rightarrow D^{(*)} N \bar{N}^{\prime} X$ processes would further question the traditional $B\left(\Lambda_{c} \rightarrow p K^{-} \pi^{+}\right)$determinations. The next section outlines briefly some methods that allow the determination of $B\left(\Lambda_{c} \rightarrow p K^{-} \pi^{+}\right)$from existing or soon available data samples.

\section{ON DETERMINING $B\left(\Lambda_{c} \rightarrow f\right)$}

This section lists a few methods that allow the determination of $B\left(\Lambda_{c} \rightarrow f\right)$, where $f$ denotes an exclusive $\left(p K^{-} \pi^{+}, \Lambda \pi, \ldots\right)$ or semi-inclusive $(\Lambda X, p X, \ldots) \Lambda_{c}$-mode.

\section{A. Method (a):}

At $e^{+} e^{-}$or $p \bar{p}$ colliders, produce $\Lambda_{c} \bar{\Lambda}_{c}$ pairs at threshold. Fully reconstruct one of the charmed baryons. Then one determines $B\left(\Lambda_{c} \rightarrow f\right)$, by measuring the probability for the remaining $\Lambda_{c}$ to be seen in $f$ [26].

\section{B. Method (b):}

At fixed target experiments, the production asymmetry can be used to determine $B\left(\Lambda_{c} \rightarrow\right.$

f) [27]. Since the total produced number of charm quarks equals that of anticharm quarks, one obtains

$$
\begin{aligned}
N\left(\Lambda_{c}\right)-N\left(\bar{\Lambda}_{c}\right) & +N\left(\Xi_{c}, \Omega_{c}\right)-N\left(\bar{\Xi}_{c}, \bar{\Omega}_{c}\right)= \\
& =N(\bar{D})-N(D)+N\left(\bar{D}_{s}\right)-N\left(D_{s}\right),
\end{aligned}
$$

where $N$ denotes the total produced number. In the lack of a $\Xi_{c}, \Omega_{c}$ production asymmetry, the coveted absolute $B\left(\Lambda_{c} \rightarrow f\right)$ is obtained via

$$
B\left(\Lambda_{c} \rightarrow f\right)=\frac{N(f)-N(\bar{f})}{N(\bar{D})-N(D)+N\left(\bar{D}_{s}\right)-N\left(D_{s}\right)} .
$$

If a $\Xi_{c}, \Omega_{c}$ production asymmetry is observed, it can be incorporated to determine $B\left(\Lambda_{c} \rightarrow\right.$ $f)$. 


\section{Method (c):}

The probability that a leading s-quark jet hadronizes as a hyperon $[P(s \rightarrow$ hyperon $)]$ can be experimentally measured. The relevant (diquark) parameters in current simulation models could then be tuned to agree with the measurements. The simulation model then predicts the probability for charmed baryon production $\left[P\left(c \rightarrow N_{c}\right)\right]$, with uncertainties typically at the $(10-20) \%$ level. Since the charm production cross-section is known, reconstructing final states of $\Lambda_{c}(f)$ permits the extraction of $B\left(\Lambda_{c} \rightarrow f\right)$.

\section{Method (d):}

At an $\Upsilon(4 S)$ factory, reconstruct a $\bar{c}$-hadron $\left[D^{(*)-}, \bar{D}^{0}, \bar{\Lambda}_{c}\right]$ with sufficiently high momentum from the continuum to remove the $B \bar{B} \rightarrow \bar{c} X$ background. contains in general a $c$-hadron. Determine the number of events that an antiproton is made in the "opposite" hemisphere $N[\bar{c} \bar{p}]$. This $\bar{p}$ cannot be a decay product of the $c$-hadron and indicates $c$-baryon production in the "opposite" hemisphere. The observation of $\Lambda_{c}$-modes $f$ in the "opposite" hemisphere allows the measurement of $N[\bar{c} \bar{p} f]$ and of

$$
B\left(\Lambda_{c} \rightarrow f\right) \approx \frac{N[\bar{c} \bar{p} f]}{N[\bar{c} \bar{p}]}
$$

A few corrections and comments must be made before this method becomes promising.

While the existence of an "opposite" hemisphere $\bar{p}$ indicates $c$-baryon production, one must correct for the fraction of the time the $\bar{c} \bar{p}$-correlation occurs with $c$-meson production. That correction can be determined by measuring the $c$-meson yield in the "opposite" hemisphere, $N\left[\bar{c} \bar{p} D_{(s)}\right]$. The probability that $\bar{c} \bar{p}$ opposite hemisphere events contain $c$-baryons can thus be determined. While the dominant fraction is $\Lambda_{c}$ 's, one may wish to correct for

\footnotetext{
$\S^{\S}$ No momentum cut is required when the data is taken at the continuum below the $\Upsilon(4 S)$ resonance.
} 
the much smaller $\Xi_{c}, \Omega_{c}$ yields. ${ }^{* * *}$

It appears that this method may not yet be feasible, because of the poor statistics for the various triple correlations $\left[\bar{c} \bar{p} f, \bar{c} \bar{p} D_{(s)}, \bar{c} \bar{p}\left\{\Xi_{c}, \Omega_{c}\right\}\right]$. It is thus important to note that much larger statistics are involved in "single" hemisphere $\bar{p} f, \bar{p} D_{(s)}, \bar{p}\left\{\Xi_{c}, \Omega_{c}\right\}$ events. The existence of a $\bar{c}$-hadron (normally) in the other hemisphere can be inferred from the reconstructed $c$-hadron [29]. Thus, the poor statistics of the triple correlations can be avoided, and $B\left(\Lambda_{c} \rightarrow f\right)$ can be measured [29. ITT

\section{E. Method (e):}

This method requires a superb vertex detector. After selecting a $b$-sample, the sample of fragmentation $\bar{p}$ 's, which originate from the interaction point and are close to the $b$-jet, indicate $b$-baryon production. (Below we discuss how to correct for $b$-meson production in association with such $\bar{p}$ 's.) Observe also negative leptons $\ell^{-}$with high $p_{T}$ and significant impact parameter, which normally are primary decay products of $b$-decays. The produced number of such $\bar{p} \ell^{-}$correlations $N\left[\bar{p} \ell^{-}\right]$is proportional to

$$
N\left[\bar{p} \ell^{-}\right] \sim P(\ldots \rightarrow \bar{p}) P\left(b \rightarrow \Lambda_{b}\right) B\left(\Lambda_{b} \rightarrow \Lambda_{c} X \ell \nu\right)
$$

where we assumed that semileptonic $\Lambda_{b}$ decays are almost always accompanied by a $\Lambda_{c}$, apart from tiny $\Lambda_{b} \rightarrow\left\{\Xi_{c} K X, D^{(*)} N X, \ldots\right\} \ell \nu$ and $b \rightarrow u$ processes. The tiny processes are at most at the $10 \%$ level of inclusive semileptonic $\Lambda_{b}$ decays, as can be inferred from an

${ }^{* * *}$ The $\Xi_{c}$ and $\Omega_{c}$ yields can be measured. Rates of specific $\Xi_{c}$ and $\Omega_{c}$ modes are related to specific $\Lambda_{c}$-modes by the SU(3)-flavor symmetry [28]. The rates of those specific modes are normally measured well with respect to the calibrating modes. Thus, the fraction of $\Lambda_{c}, \Xi_{c}, \Omega_{c}$ in $\bar{c} \bar{p}$ events can be determined.

${ }^{\dagger \dagger}$ This $B\left(\Lambda_{c} \rightarrow f\right)$ measurement neglects long range $c \bar{c}$ production correlations, which are expected to be small. They can be accounted for in triple correlation studies. 
analogy to semileptonic $\bar{B}$-decay measurements [30,31. Ratios of specific $\Lambda_{c}$ decay rates can thus be determined

$$
\frac{\Gamma\left(\Lambda_{c} \rightarrow f^{\prime}\right)}{\Gamma\left(\Lambda_{c} \rightarrow f\right)}=\frac{N\left[\bar{p} \ell^{-} f^{\prime}\right]}{N\left[\bar{p} \ell^{-} f\right]}
$$

where $f, f^{\prime}=p K \pi, \Lambda n \pi, \Lambda X, p X, \ldots$. Information concerning semi-inclusive $\Lambda_{c}$ decay rates can thus be obtained. Even absolute $\Lambda_{c}$ branching ratios can be determined via

$$
B\left(\Lambda_{c} \rightarrow f\right) \approx \frac{N\left[\bar{p} \ell^{-} f\right)}{N\left[\bar{p} \ell^{-}\right]}
$$

One must correct for the fraction of the time the $b$-jet nearby the fragmentation $\bar{p}$ gives rise to a $b$-meson (rather than a $b$-baryon). The correction factor can be obtained in several ways:

1. Measure the probability that $\bar{p} \ell^{-}$events involve (detached) charmed mesons, which together with the $\ell^{-}$point to the $b$-decay vertex.

2. Select a charged $B^{-}$sample by using vertex charge and vertex mass. Study the fraction of the time this $B^{-}$sample involves a nearby fragmentation $\stackrel{(-)}{p}$.

3. In the $b$-enriched sample with one fragmentation $\bar{p}$, observe an additional fragmentation $p$ also from the interaction point and nearby the $b$-jet. Since baryon number is conserved, it is likely a $b$-meson was produced. (The possibility of more than one nearby baryon-antibaryon pair production may not be negligible, however.) Because isospin symmetry allows one to determine the production ratio of fragmentation neutrons versus protons, the fraction of the time $b$-mesons are made can be inferred.

It may prove useful to introduce stringent cuts on the fragmentation $\bar{p}$, so as to reduce the $b$-meson fraction.

\section{F. Method (f):}

If it were possible to theoretically relate $\Gamma\left(\Lambda_{b} \rightarrow \Lambda J / \psi\right)$ to $\Gamma\left(\bar{B} \rightarrow \bar{K}^{(*)} J / \psi\right)$ then the observed $\Lambda J / \psi$ sample of fully reconstructed $\Lambda_{b}$ decays permits the determination of 
$P\left(b \rightarrow \Lambda_{b}\right)$. Once $P\left(b \rightarrow \Lambda_{b}\right)$ is known, then the $\Lambda_{c}[\rightarrow f] \ell^{-}$sample yields $B\left(\Lambda_{c} \rightarrow f\right)$.

\section{G. Conclusion}

Those are then some suggestions to determine $B\left(\Lambda_{c} \rightarrow f\right)$. Undoubtedly, many possible variations and improvements will become obvious to the dedicated experimenter. Appendix A sketches a determination of $B\left(\Lambda_{b} \rightarrow X \ell \nu\right)$ and $\left|V_{c b}\right|$ with a reduced dependence on $B\left(\Lambda_{c} \rightarrow\right.$ $f)$.

\section{IMPLICATIONS}

If $B\left(\Lambda_{c} \rightarrow p K^{-} \pi^{+}\right)$turns out to be significantly larger than the current world average, as we predict, then there will be many ramifications. Some of them are:

- Since $B\left(\Lambda_{c} \rightarrow p K^{-} \pi^{+}\right)$normalizes most heavy baryon productions and decays, the heavy baryon decay tables listed in Ref. [7] will have to be recalibrated accordingly.

- The $\Lambda_{b}: \bar{B}: \bar{B}_{s}$ production fractions will be affected. The $\Lambda_{b}$ fraction will be reduced sizably from current estimates while the $\bar{B}$ and $\bar{B}_{s}$ fractions will increase. The measured $\Lambda_{b}$ branching ratios thus increase sizably, while the $B_{(s)}$ ones decrease.

- The number of charms per $b$-decay decreases on two counts. First, the $\Xi_{c}$ yield in $\bar{B}$ decays is predicted to be sizably lower than its measured central value. Second, the $B\left(\Lambda_{c} \rightarrow p K^{-} \pi^{+}\right)$is significantly larger than expected, resulting into a lower $\Lambda_{c}$ yield in $b$ decays than presently believed. Quantitative estimates can be found in Refs. [9, 32].

- The charmless yield in $B$-meson decays is larger than a recent indirect extraction [17]. CLEO [17] measured the flavor-specific charm yields in $B$ decays in a way that removes the large systematic uncertainty due to $B\left(D^{0} \rightarrow K^{-} \pi^{+}\right)$That beautiful

\footnotetext{
${ }_{\ddagger \ddagger}$ That study enables Appendix B to point out a complementary method for determining $B\left(D^{0} \rightarrow\right.$
} 
analysis then assumed that the inclusive baryon yield in $\bar{B}$ decays is saturated by weakly decaying charmed baryon production $\left(N_{c}\right)$, resulting in $B(b \rightarrow$ no open $c)=$ $0.04 \pm 0.04$. In contrast, this note questions the validity of the assumption and uses $B\left(\bar{B} \rightarrow N_{c} X\right)=0.032 \pm 0.011$, which implies a larger $B(b \rightarrow$ no open $c)=0.07 \pm 0.04$. Table II summarizes both viewpoints.

Those are then some of the consequences of our view of heavy baryon production and decay.

\section{CONCLUSIONS}

Because the $\Lambda_{c} \rightarrow p K^{-} \pi^{+}$process normalizes heavy baryon production and decay, its absolute branching ratio must be known to the highest accuracy achievable. Analyses of baryon production in $B$ meson decays dominate the traditional $B\left(\Lambda_{c} \rightarrow p K^{-} \pi^{+}\right)$"measurements",

$$
B\left(\Lambda_{c} \rightarrow p K^{-} \pi^{+}\right)= \begin{cases}0.044 \pm 0.006 & {[7]} \\ 0.027 \pm 0.005\end{cases}
$$

Those analyses however made several questionable assumptions, summarized in Table 1. Instead, a considerably larger $B\left(\Lambda_{c} \rightarrow p K^{-} \pi^{+}\right)$emerges [11,9, 33],

$$
B\left(\Lambda_{c} \rightarrow p K^{-} \pi^{+}\right)=0.07 \pm 0.02
$$

by combining theory and available experimental data on semileptonic charm transitions.

This confusing state of affairs can be clarified by searching for and observing the traditionally overlooked $\bar{B} \rightarrow D^{(*)} N \bar{N}^{\prime} X$ processes. We predict those $\bar{B} \rightarrow D^{(*)} N \bar{N}^{\prime} X$ processes to constitute a sizable fraction of all $\bar{B} \rightarrow$ baryons transitions. They should be observable in existing data samples. If this is borne out, then $B\left(\Lambda_{c} \rightarrow p K^{-} \pi^{+}\right)$must be determined afresh.

$\left.K^{-} \pi^{+}\right)$, which does not involve the soft pion from $D^{*+} \rightarrow \pi^{+} D^{0}$ transitions. The result is $B\left(D^{0} \rightarrow\right.$ $\left.K^{-} \pi^{+}\right)=0.035 \pm 0.002$, by equating two CLEO measurements for $Y_{D}$. 
That can be accomplished in a variety of methods, some of which Section 4 briefly outlined. A considerably larger $B\left(\Lambda_{c} \rightarrow p K^{-} \pi^{+}\right)$than currently accepted will have ramifications, such as:

- The heavy baryon decay tables will have to be recalibrated.

- The $b \rightarrow \Lambda_{b}$ production fraction decreases.

- The measured number of charm per $b$-decay decreases.

- The $\bar{B} \rightarrow$ baryons transitions are more involved than currently modeled.

More theoretical and experimental investigations are highly welcome, as it will improve our understanding of heavy baryon production and decay.

\section{ACKNOWLEDGMENTS}

We thank M. Barnett for encouraging us to write up this note. We are grateful to M.S. Alam, M. Beneke, D.Z. Besson, G. Buchalla, P. Burchat, R.N. Cahn, H.W.K. Cheung, Su Dong, O. Hayes, N. Isgur, M. Luke, M. Paulini, J.L. Rosner, I. Shipsey, N.G. Uraltsev, M. Voloshin and C. Wohl for discussions. This work was supported in part by the Department of Energy, Contract No. DE-AC02-76CH03000.

\section{APPENDIX A: ON DETERMINING $B\left(\Lambda_{b} \rightarrow X \ell \nu\right)$ AND $\left|V_{c b}\right|$ WITH A REDUCED $B\left(\Lambda_{c} \rightarrow f\right)$ UNCERTAINTY.}

At present there exists a discrepancy between theory and experiment concerning the lifetime ratio $\tau\left(\Lambda_{b}\right) / \tau\left(B_{d}\right)[7]$. The most plausible explanation is an enhanced nonleptonic $\Lambda_{b}$-rate, without a corresponding enhancement in the semileptonic $\Lambda_{b}$-rate. This Appendix discusses one way to determine the semileptonic $\Lambda_{b}$ rate and branching ratio, thereby probing

the underlying cause of the lifetime discrepancy. Building on the discussion presented in 
method (e) of Section 4, one notes that the produced number of $\ell^{-} f$ events is proportional to

$$
N\left[\ell^{-} f\right] \sim P(\ldots \rightarrow b) P\left(b \rightarrow \Lambda_{b}\right) B\left(\Lambda_{b} \rightarrow \Lambda_{c} X \ell \nu\right) B\left(\Lambda_{c} \rightarrow f\right)
$$

where it is understood that the various backgrounds have been corrected for. In addition, the direct production of $\Lambda_{c}$ baryons can be studied as well. To remove the large $b \rightarrow \Lambda_{c}$ background, it may prove advantageous to focus on the high momentum $\Lambda_{c} \rightarrow f$ sample,

$$
N[f] \sim P(\ldots \rightarrow c) P\left(c \rightarrow \Lambda_{c}\right) B\left(\Lambda_{c} \rightarrow f\right) .
$$

The production ratio $P(\ldots \rightarrow b) / P(\ldots \rightarrow c)$ is well known, and HQET can in principle determine the ratio

$$
\frac{P\left(b \rightarrow \Lambda_{b}\right)}{P\left(c \rightarrow \Lambda_{c}\right)}=1+\mathcal{O}\left(1 / m_{Q}\right)
$$

The semileptonic $\Lambda_{b}$-branching ratio can thus be determined

$$
B\left(\Lambda_{b} \rightarrow X \ell \nu\right) \approx B\left(\Lambda_{b} \rightarrow \Lambda_{c} X \ell \nu\right) \sim \frac{N\left[\ell^{-} f\right]}{N[f]}
$$

Even the CKM parameter $\left|V_{c b}\right|$ can be extracted by measuring the exclusive $\Lambda_{b} \rightarrow \Lambda_{c} \ell \nu$ branching ratio, $B\left(\Lambda_{b} \rightarrow \Lambda_{c} \ell \nu\right)$. That measurement combined with $\tau\left(\Lambda_{b}\right)$ determines $\Gamma\left(\Lambda_{b} \rightarrow \Lambda_{c} \ell \nu\right)$. HQET [3,2] and lattice studies [34] inform on the relevant form-factors, so that $\left|V_{c b}\right|$ can be determined from that $\Lambda_{b} \rightarrow \Lambda_{c} \ell \nu$ measurement.

\section{APPENDIX B: ON THE $B\left(D^{0} \rightarrow K^{-} \pi^{+}\right)$VALUE}

Not only does $B\left(D^{0} \rightarrow K^{-} \pi^{+}\right)$calibrate most other $D^{0}$ decays, but the $D^{+}$and $D_{s}^{+}$ calibration-modes $\left[D^{+} \rightarrow K^{-} \pi^{+} \pi^{+}\right.$and $\left.D_{s}^{+} \rightarrow \phi \pi^{+}\right]$are tied to it as well [7]. The $D^{0} \rightarrow$ $K^{-} \pi^{+}$process normalizes charmed meson production and decay, in analogy to the role of

\footnotetext{
$\S \S \S$ It would be useful to calculate the momentum dependence of this ratio.
} 
the $\Lambda_{c} \rightarrow p K^{-} \pi^{+}$transition for charmed baryon studies. This Appendix introduces one more method for determining $B\left(D^{0} \rightarrow K^{-} \pi^{+}\right)$, which yields

$$
B\left(D^{0} \rightarrow K^{-} \pi^{+}\right)=0.035 \pm 0.002 .
$$

There exist now two CLEO measurements of $D$ production in flavor-blind $B$ decays,

$$
Y_{D} \equiv B(\bar{B} \rightarrow D X)+B(\bar{B} \rightarrow \bar{D} X) .
$$

The first is a high statistics measurement of inclusive $D$ and $\bar{D}$ production in $B$ decays, which is inversely proportional to $B\left(D^{0} \rightarrow K^{-} \pi^{+}\right)$[22],

$$
Y_{D}=(0.876 \pm 0.037)\left[\frac{0.0388}{B\left(D^{0} \rightarrow K^{-} \pi^{+}\right)}\right] .
$$

The second is not sensitive to $B\left(D^{0} \rightarrow K^{-} \pi^{+}\right)$,

$$
Y_{D}=0.96 \pm 0.05
$$

and was deduced from Ref. [17] as discussed below. Equating the two and solving for $B\left(D^{0} \rightarrow K^{-} \pi^{+}\right)$yields (B1). This value agrees with the one obtained in Refs. [9, 35, 36, and is somewhat below the world average [37], $B\left(D^{0} \rightarrow K^{-} \pi^{+}\right)=0.0388 \pm 0.0010$.

The world average is dominated by studies involving the soft $\pi^{+}$in $D^{*+} \rightarrow \pi^{+} D^{0}$ decays, which require the accurate modeling of the tails of the soft pion momentum spectrum. Because such accurate modeling may prove more difficult than presently appreciated [36], measurements of $B\left(D^{0} \rightarrow K^{-} \pi^{+}\right)$insensitive to such soft $\pi^{+}$'s should also be pursued. Such methods were discussed in the literature [9, 36, 17]. This Appendix introduces yet another one.

Eq. (B4) is obtained via

$$
Y_{D}=D_{\ell} \times\left(1+r_{D}\right) \times L .
$$

Here theory delivers [17]

$$
L \equiv B(\bar{B} \rightarrow D X \ell \nu) / B(\bar{B} \rightarrow X \ell \nu)=0.97 \pm 0.02,
$$


while the other quantities were measured [17]:

$$
\begin{gathered}
D_{\ell} \equiv \frac{B(\bar{B} \rightarrow D X)}{B(\bar{B} \rightarrow D X \ell \nu) / B(\bar{B} \rightarrow X \ell \nu)}=0.901 \pm 0.037 \\
r_{D} \equiv B(\bar{B} \rightarrow \bar{D} X) / B(\bar{B} \rightarrow D X)=0.100 \pm 0.031
\end{gathered}
$$

Note that several uncertainties cancel in the $D_{\ell} \times\left(1+r_{D}\right)$ combination [38], so that CLEO can determine $Y_{D}\left[B\left(D^{0} \rightarrow K^{-} \pi^{+}\right)\right]$with a smaller error than given in Eq. (B4) [(B1)]. 


\section{TABLES}

TABLE I. The conventional determination of $B\left(\Lambda_{c} \rightarrow p K^{-} \pi^{+}\right)$involves $\bar{B} \rightarrow$ baryons analyses with the assumptions tabulated here.

\begin{tabular}{|l|l|}
\hline \hline Assumptions & Comments \\
\hline \hline$B\left(\bar{B} \rightarrow D^{(*)} N \bar{N}^{\prime} X\right)=0$ & $\begin{array}{l}\text { Could be } \sim \text { few \%. Theoretical Dalitz plot allows for } \\
\text { sizable } \bar{B} \rightarrow D^{(*)} N \bar{N}^{\prime} X .\end{array}$ \\
\hline$B\left(\bar{B} \rightarrow \Xi_{c} X, \Omega_{c} X\right)=0$ & $\bar{B} \rightarrow \Xi_{c} X$ large \\
\hline$B(\bar{B} \rightarrow$ baryons $)=0.068 \pm 0.006$ & $\begin{array}{l}\text { Value questionable, because derived under the assumption } \\
\text { that } B\left(\bar{B} \rightarrow D^{(*)} N \bar{N}^{\prime} X\right)=0 . \text { Instead, this note }\end{array}$ \\
& determines model-independent lower limits \\
& from existing light baryon yields. \\
\hline
\end{tabular}

TABLE II. Open charm production in $\bar{B}$ decays. The last row lists the charmless yield which is obtained via $B(b \rightarrow$ no open $c)=1-B(b \rightarrow$ open $c)$.

\begin{tabular}{|l|c|}
\hline \hline $\bar{B}[\equiv b \bar{q}] \rightarrow$ open $c$ & CLEO [17] (This note) \\
\hline $\bar{B} \rightarrow D^{0}, D^{+}$ & $0.87 \pm 0.04$ \\
\hline $\bar{B} \rightarrow D_{s}^{+}$ & $0.02 \pm 0.01$ \\
\hline $\bar{B} \rightarrow N_{c}\left[\equiv \Lambda_{c}, \Xi_{c}, \Omega_{c}\right]$ & $0.065 \pm 0.015(0.032 \pm 0.011)$ \\
\hline $\bar{B} \rightarrow$ no open $c$ & $0.04 \pm 0.04(0.07 \pm 0.04)$ \\
\hline \hline
\end{tabular}




\section{REFERENCES}

[1] N. Isgur and M.B. Wise, Nucl. Phys. B348, 276 (1991).

[2] M. Neubert, Phys. Rept. 245, 259 (1994).

[3] H. Georgi, B. Grinstein and M.B. Wise, Phys. Lett. B252, 456 (1990).

[4] For a recent review, see for instance, I. Bigi, M. Shifman and N. Uraltsev, Ann. Rev. Nucl. Part. Sci. 47, 591 (1997).

[5] R. Springer, private communication.

[6] I. Dunietz, Z. Phys. C56, 129 (1992).

[7] R.M. Barnett et al. (Particle Data Group), Phys. Rev. D54, 1 (1996).

[8] M.M. Zoeller (CLEO collaboration), Ph. D. Thesis, submitted to the State University of New York, Albany, 1994.

[9] I. Dunietz, Fermilab report, FERMILAB-PUB-96-104-T, June 1996 hep-ph/9606247.

[10] H. Albrecht et al. (ARGUS Collaboration), Z. Phys. C56, 1 (1992).

[11] T. Bergfeld et al. (CLEO Collaboration), Phys. Lett. B323, 219 (1994).

[12] A. Dighe and I. Dunietz, unpublished.

[13] I.I. Bigi, N.G. Uraltsev, A.I. Vainshtein, Phys. Lett. B293, 430 (1992); (E) Phys. Lett. B297, 477 (1993); A.V. Manohar and M.B. Wise, Phys. Rev. D49, 1310 (1994); I.I. Bigi, UND-HEP-95-BIG02, June 1995 hep-ph/9508408.

[14] Y. Kubota et al. (CLEO collaboration), Phys. Rev. D54, 2994 (1996).

[15] M.B. Voloshin, Phys. Lett. B385, 369 (1996).

[16] G. Crawford et al. (CLEO collaboration), Phys. Rev. D45, 752 (1992).

[17] T.E. Coan et al. (CLEO collaboration), Report number CLNS-97-1516, October 1997, 
hep-ex/9710028.

[18] M. Paulini, for the ARGUS collaboration, Proceedings of the XXVIIIth Recontre de Moriond "Electroweak Interactions and Unified Theories"; Les Arcs, France 1993; ed. J. Trân Thanh Vân, Editions Frontiers, 1993, p. 253.

[19] I. Dunietz, P.S. Cooper, A.F. Falk, and M.B. Wise, Phys. Rev. Lett. 73, 1075 (1994).

[20] D. Cinabro et al. (CLEO collaboration), Cornell report, CLEO CONF 94-8, 1994.

[21] T.E. Browder, K. Honscheid and D. Pedrini, Annual Review of Nuclear and Particle Science 46, 395 (1996).

[22] L. Gibbons et al. (CLEO collaboration), Phys. Rev. D56, 3783 (1997).

[23] M. Peshkin and J.L. Rosner, Nucl. Phys. B122, 144 (1977).

[24] P.L. Tipton (CLEO collaboration), Ph. D. Thesis, University of Rochester report, UR 984, 1987; M.S. Alam et al. (CLEO collaboration), Phys. Rev. Lett. 58, 1814 (1987).

[25] G. Bonvicini et al. (CLEO collaboration), CLNS-97-1519, Dec. 1997, hep-ex/9712008.

[26] G.S. Abrams et al. (Mark II collaboration), Phys. Rev. Lett. 44, 10 (1980).

[27] H.W.K. Cheung, private communication.

[28] A. Datta, I. Dunietz and P.J. O’Donnell, in preparation.

[29] I. Dunietz and J.L. Lewis, in preparation.

[30] D. Buskulic et al. (ALEPH Collaboration), Z. Phys. C73, 601 (1997).

[31] N. Isgur, in progress.

[32] I. Dunietz, J. Incandela, F.D. Snider and H. Yamamoto, Eur. Phys. J. C1, 211 (1998).

[33] I. Dunietz, in the proceedings of the 2nd International Conference on B Physics and CP Violation, Honolulu, HI, 24-28 Mar 1997, FERMILAB-CONF-97-230-T, hep- 
ph/9708252.

[34] K.C. Bowler et al., EDINBURGH-96-31, Sep 1997, hep-lat/9709028.

[35] I. Dunietz, J. Incandela, F. D. Snider, K. Tesima, I. Watanabe, FERMILAB-PUB-96026-T, June 1996, hep-ph/9606327.

[36] I. Dunietz, J. Incandela and F.D. Snider, Nucl. Phys. Proc. Suppl. 55A, 145 (1997).

[37] J.D. Richman, in proceedings of the 28th International Conference on High Energy Physics, Warsaw, Poland, July 1996, hep-ex/9701014.

[38] We thank E. Thorndike for discussions on this point. 


\section{FIGURES}

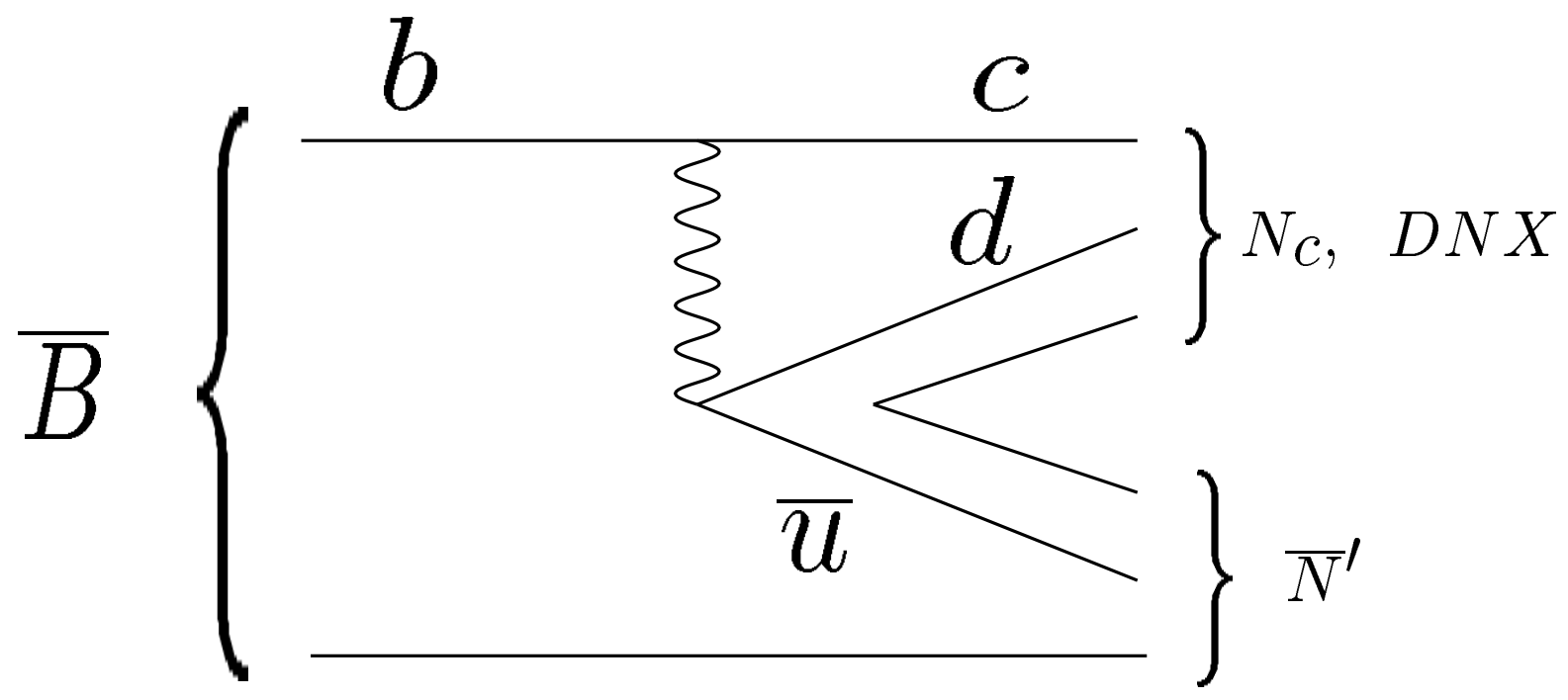

FIG. 1. Graph depicting baryon production in $\bar{B}$ decays.

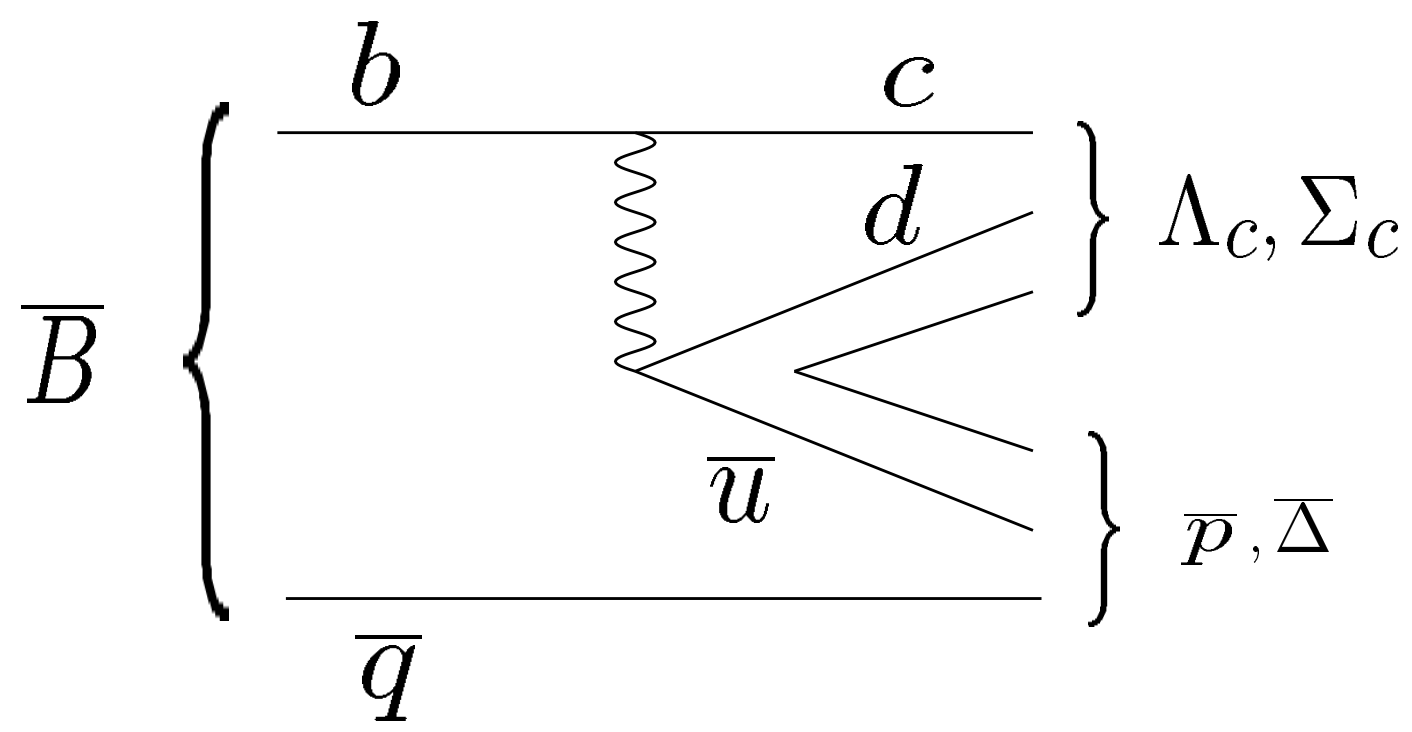

FIG. 2. Graph responsible for the two-body $\bar{B}$-modes, $\bar{B} \rightarrow\left\{\Lambda_{c}, \Sigma_{c}\right\}\{\bar{p}, \bar{\Delta}\}$. 


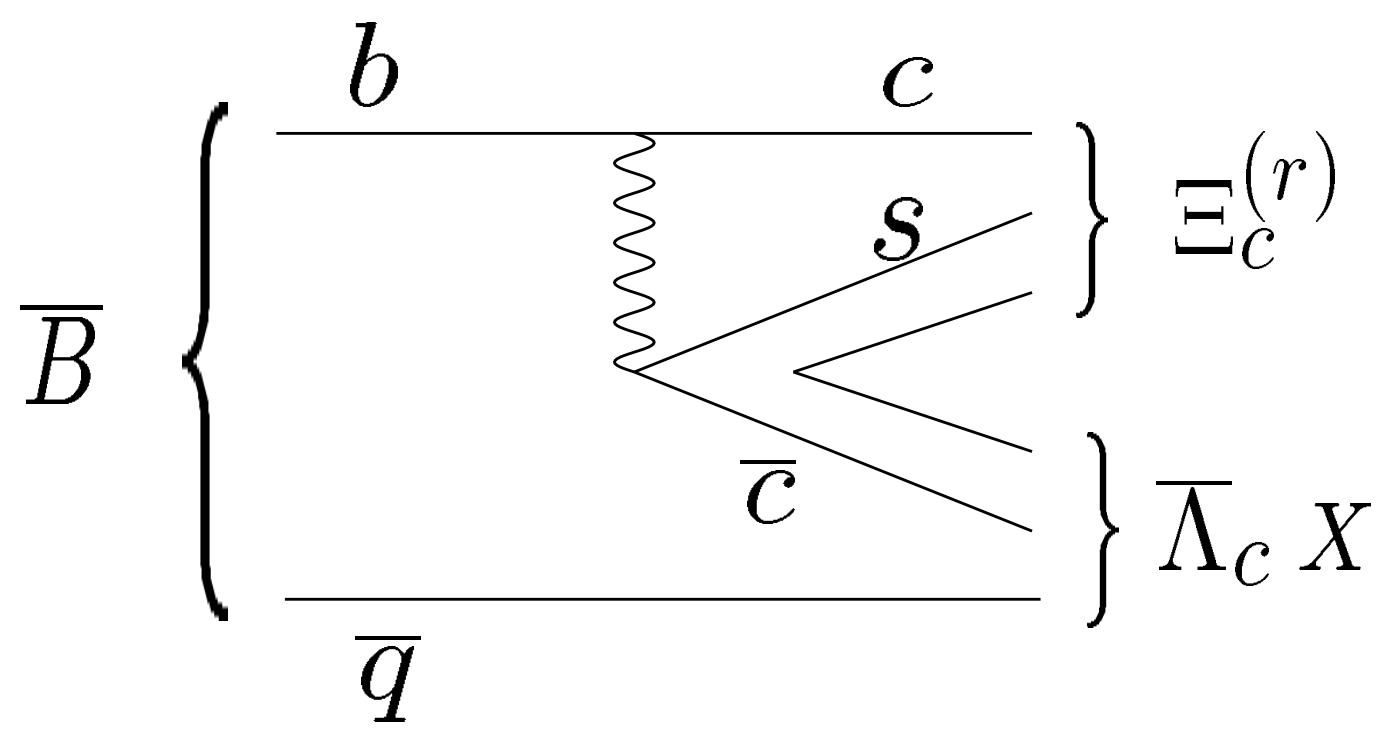

FIG. 3. Graph governing $\bar{B} \rightarrow \Xi_{c}^{(r)} \bar{\Lambda}_{c} X$ transitions.

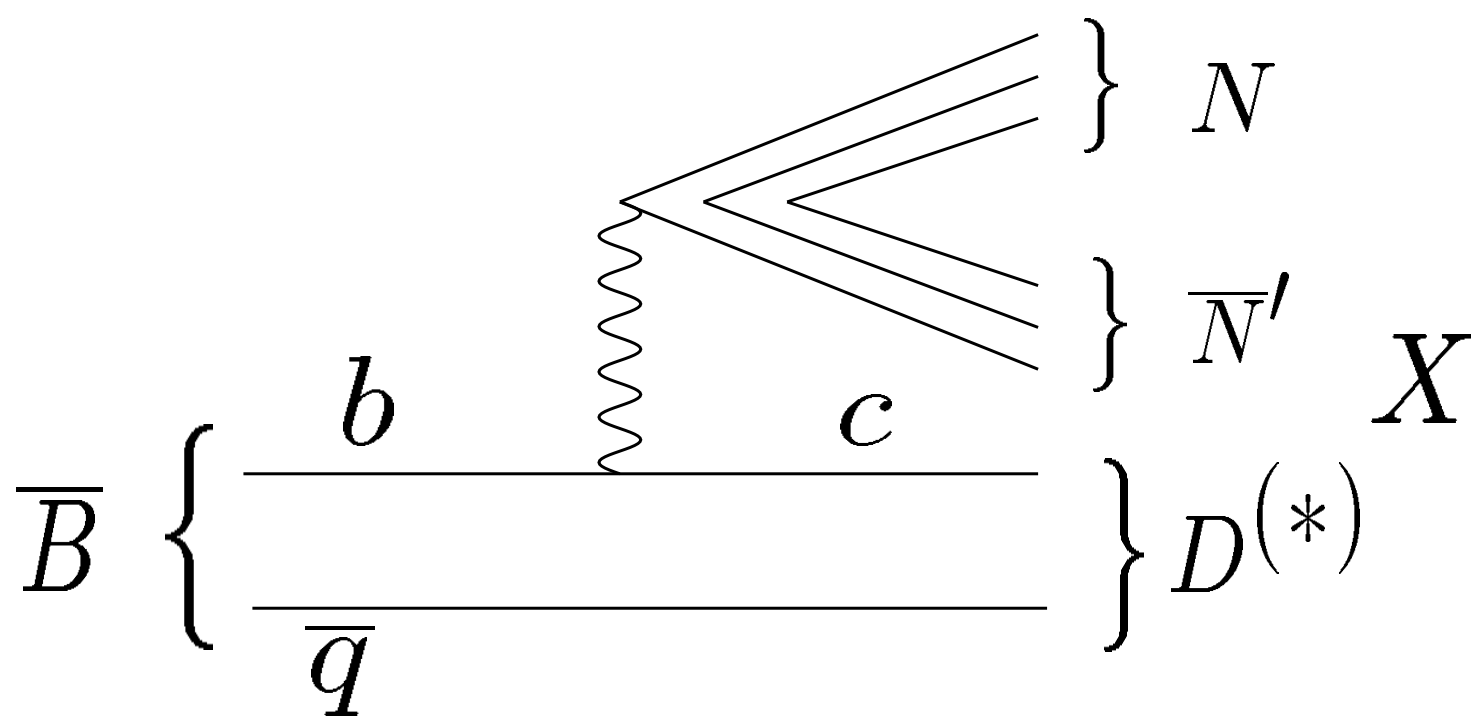

FIG. 4. Baryon production in $\bar{B}$ decays, wherein the virtual $W$ hadronizes into a baryonantibaryon pair. 